\title{
The bryophyte flora of Lågskär - an isolated island in the Baltic Sea
}

\author{
Anna Oldén \\ Department of Biological and Environmental Science, University of Jyväskylä, \\ P.O. Box 35, FI-40014 University of Jyväskylä, Finland \\ E-mail: anna.m.olden@jyu.fi
}

\begin{abstract}
This study reports the bryophyte flora of Lågskär, an isolated lighthouse island in the Åland province of Finland. A total of 81 bryophyte species were found on Lågskär, including 74 moss species and seven liverwort species. Syntrichia ruraliformis is reported for the first time in Finland, whereas Ceratodon conicus was believed to be extinct in Finland and Brachythecium turgidum was considered regionally extinct from southern Finland.
\end{abstract}

\section{INTRODUCTION}

Lågskär is an old lighthouse island in the northern Baltic Sea, in Finland's Åland archipelago. The weather conditions are harsh but the island still supports very diverse vegetation. Lågskär is located far from other islands with vegetation and therefore the sea forms a distinctive barrier for dispersal. The species composition and biogeography of the island is interesting due to the remote location and harsh conditions. Compared to the Finnish mainland the island has a southern location and an oceanic climate and therefore the species communities on the island could be more similar to those in Sweden or Estonia. The aim of this study was to record the bryophyte flora (mosses and liverworts) of Lågskär.

\section{Study area}

Lågskär belongs to the Lemland municipality in Åland. It is located at 59 50 '23" N and $19^{\circ} 55^{\prime} 42^{\prime \prime}$ $\mathrm{E}$, which is approximately $19.5 \mathrm{~km}$ south of the Åland main island, $140 \mathrm{~km}$ west of the Finnish mainland, $48 \mathrm{~km}$ east of Sweden and $174 \mathrm{~km}$ north-west of the Estonian island Hiiumaa (Fig. 1). The nearest forested island is located 13.5 $\mathrm{km}$ north of Lågskär. The average annual precipitation in the area is $650-700 \mathrm{~mm}$, the mean temperature in January is $-1^{\circ} \mathrm{C}$ and the mean temperature in July is $17^{\circ} \mathrm{C}$. It is very windy on the island and because the sun shines freely onto the majority of the island surface, the microclimate is mostly dry. The island is located in the hemiboreal vegetation zone (Ahti et al. 1968). The land area of the island is $0.43 \mathrm{~km}^{2}$. Lågskär

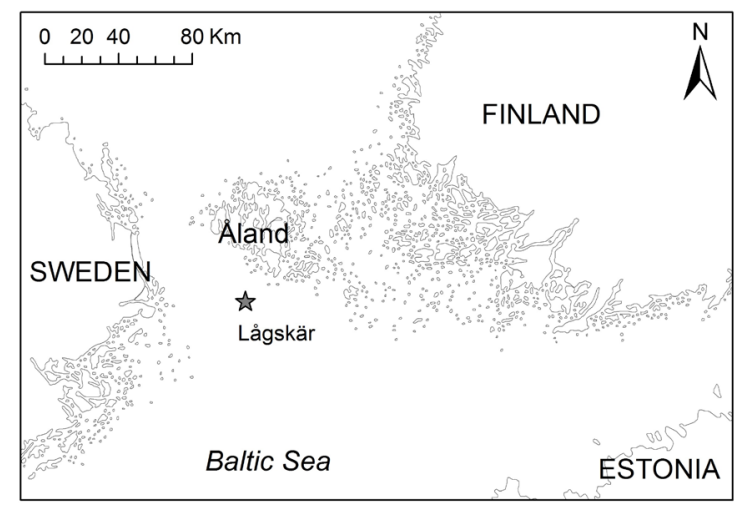

Fig. 1. The location of Lågskär in the Baltic Sea. (C) National Land Survey of Finland, 2010.

is not officially protected but it is an Important Bird Area and it is a part of a Ramsar site.

Most of the island consists of bare acidic bedrock. Vegetation occurs mostly in the depressions and cracks of the rock (Fig. 2). Soil deposits in the depressions are oligotrophic, mesotrophic or in some patches eutrophic. The vegetation in the depressions is very diverse and varied, including for example Achillea millefolium, Allium schoenoprasum, Galium spp., Plantago spp., Sedum spp. and graminoids. In larger depressions there are patches of oligotrophic or mesotrophic bogs and fens as well as moist eutrophic herbrich forests. Dominant plants in the bogs are Calluna vulgaris, Vaccinium uliginosum, Rubus chameamorus, Eriophorum spp. and Carex spp. 


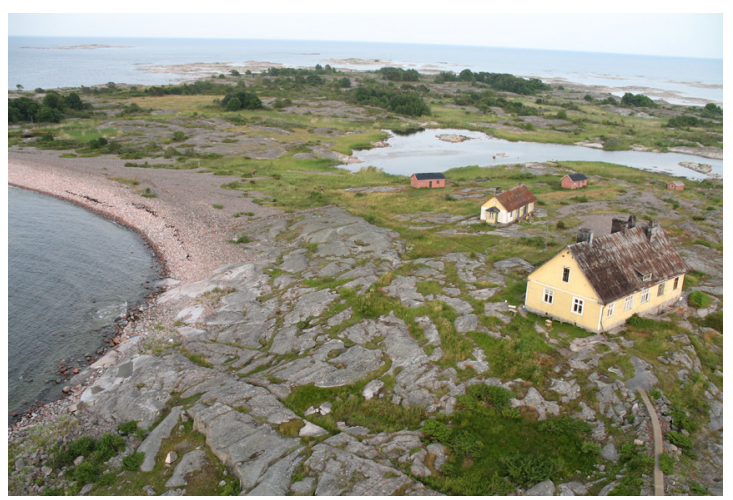

Fig. 2. A view of Lågskär island, photographed from the lighthouse towards south-east. Rocks and trampled surroundings of the buildings can be seen in the front, shore meadows around the bay, dry meadows in the central part of the island, and scattered patches of forest near the central parts.

In the forest patches the dominant trees are $A l$ nus glutinosa, Picea abies, Sorbus aucuparia and $S$. hybrida, and other common plants include Athyrium filix-femina, Cornus suecica, Dryopteris carthusiana, Filipendula ulmaria, Geum rivale and Ribes alpinum. The dry, sandy areas in the center of the island are dominated by meadow vegetation (e.g. Fragaria vesca, Hypericum perforatum, Vicia cracca and graminoids) and especially junipers (Juniperus communis) and sea-buckthorn (Hippophaë rhamnoides). There are also relatively large areas of moist meadows and shore meadows on the island (including e.g. Juncus spp., Lysimachia vulgaris, Lythrum salicaria, Ophioglossum vulgatum and Triglochin maritima), but they are mostly overgrown with the common weed (Phragmites australis).

Lågskär is situated near many important Baltic shipping routes and it is therefore an important lighthouse location. The first lighthouse was built in 1696 and since then there have been four lighthouses on the north-western part of the island, called Hemlandet. The island was populated by lighthouse managers, their families and their livestock until 1961 when the lighthouse became automated. The livestock grazed freely on the island, keeping the meadows open. Winter fodder, firewood and edible produce were also collected form the meadows and the forests. After the automation of the lighthouse the buildings on the island have been used mostly by ornithologists (Laaksonen 2004). The human impact on the island is nowadays low, including mostly trampling near the buildings on Hemlandet and elsewhere on paths. During recent years a part of the dry, overgrown meadow in the central part of the island has been managed by clearing junipers and mowing.

\section{MATERIAL AND METHODS}

The bryophyte flora of Lågskär was studied in July 2011. All of the habitat types on the island (trampled yard area and paths, dry meadows, shore meadows, forests, bogs and fens, rocks) were surveyed and bryophytes growing on all substrates were recorded. Specimens were collected of species that could not be identified in the field and they were later identified under the microscope. Specimens of noteworthy species have been deposited in the Natural History Collection of Jyväskylä University Museum (JYV). The nomenclature of bryophytes follows Ulvinen and Syrjänen (2009) and the classification of red-listed species follows Syrjänen et al. (2010).

\section{RESULTS}

A total of 81 bryophyte species were found on Lågskär, including 74 moss species and seven liverwort species. All the observed species are listed in Table 1 with the authors of the scientific names. The table also provides their status in Finland and in Åland before the species were observed in Lågskär (Sammaltyöryhmä 2011) as well as currently (Sammaltyöryhmä 2014), and the occurrence of the species on the main habitat types of the island.

The observed species include 14 noteworthy species, which is $17 \%$ of all observed species on Lågskär. Syntrichia ruraliformis was recorded for the first time in Finland, and it is currently classified as data deficient in the country. It was found in three locations around the lighthouse, growing on gravel and on weathered, sandy concrete on the footing of the lighthouse. Another moss species, Ceratodon conicus, that was believed to have gone extinct in Finland, was found growing in a crack in the rock near the main building. It is now classified as critically endangered in Finland. Brachythecium turgidum, that was believed to have gone regionally extinct in southern Finland, was observed growing on 
Table 1. The species observed in Lågskär, with their status in Finland and in the province of Åland before the Lågskär observations (Sammaltyöryhmä 2011) and currently (Sammaltyöryhmä 2014), and their occurrence of the species on the main habitat types of the island $(1=$ observed at least once, empty $=$ not observed).

Status in Finland:

RE Regionally extinct

CR Critically endangered

NT Nearly threatened

DD Data deficient

LC Least concern

NEW Not observed in the country before
Status in Åland:

RE Regionally extinct

RT Regionally threatened

IND Indicator species

- Not observed in the province since 1940

- Common

\begin{tabular}{|c|c|c|c|c|c|c|c|c|}
\hline Species & $\begin{array}{l}\text { Status } \\
2011\end{array}$ & $\begin{array}{l}\text { Status } \\
2014\end{array}$ & $\begin{array}{l}\text { Tramp- } \\
\text { led area }\end{array}$ & $\begin{array}{l}\text { Dry } \\
\text { meadows }\end{array}$ & $\begin{array}{l}\text { Shore } \\
\text { meadows }\end{array}$ & Forests & Rocks & $\begin{array}{l}\text { Bogs, } \\
\text { fens }\end{array}$ \\
\hline \multicolumn{9}{|l|}{ Mosses } \\
\hline $\begin{array}{l}\text { Abietinella abietina (Hedw.) } \\
\text { M.Fleisch. }\end{array}$ & $\mathrm{LC} / \bullet$ & $\mathrm{LC} / \bullet$ & & 1 & & & & \\
\hline $\begin{array}{l}\text { Amblystegium serpens (Hedw.) } \\
\text { Schimp. }\end{array}$ & $\mathrm{LC} / \bullet$ & $\mathrm{LC} / \bullet$ & 1 & & & 1 & 1 & \\
\hline $\begin{array}{l}\text { Aulacomnium androgynum (Hedw.) } \\
\text { Schwägr. }\end{array}$ & $\mathrm{LC} / \bullet$ & $\mathrm{LC} / \bullet$ & & & & 1 & & 1 \\
\hline $\begin{array}{l}\text { Aulacomnium palustre (Hedw.) } \\
\text { Schwägr. }\end{array}$ & $\mathrm{LC} / \bullet$ & $\mathrm{LC} / \bullet$ & & & 1 & & 1 & 1 \\
\hline Barbula convoluta Hedw. & $\mathrm{LC} / \bullet$ & $\mathrm{LC} / \bullet$ & 1 & & & & 1 & \\
\hline $\begin{array}{l}\text { Brachytheciastrum velutinum } \\
\text { (Hedw.) Ignatov \& Huttunen }\end{array}$ & $\mathrm{LC} / \bullet$ & $\mathrm{LC} / \bullet$ & & & & 1 & & \\
\hline $\begin{array}{l}\text { Brachythecium albicans (Hedw.) } \\
\text { Schimp. }\end{array}$ & $\mathrm{LC} / \bullet$ & $\mathrm{LC} / \bullet$ & 1 & 1 & & & 1 & \\
\hline $\begin{array}{l}\text { Brachythecium glareosum (Bruch ex } \\
\text { Spruce) Schimp. }\end{array}$ & $\begin{array}{l}\mathrm{LC} / \\
\text { IND }\end{array}$ & $\begin{array}{l}\mathrm{LC} / \\
\text { IND }\end{array}$ & 1 & 1 & & & & \\
\hline $\begin{array}{l}\text { Brachythecium mildeanum } \\
\text { (Schimp.) Schimp. }\end{array}$ & $\mathrm{LC} / \mathrm{O}$ & $\mathrm{LC} / \bullet$ & & & & 1 & & \\
\hline $\begin{array}{l}\text { Brachythecium rutabulum (Hedw.) } \\
\text { Schimp. }\end{array}$ & $\mathrm{LC} / \bullet$ & $\mathrm{LC} / \bullet$ & & & & 1 & & \\
\hline $\begin{array}{l}\text { Brachythecium turgidum (Hartm.) } \\
\text { Kindb. }\end{array}$ & LC/ RE & LC/ RT & & & 1 & & & \\
\hline Bryum alpinum Huds. ex With. & $\mathrm{LC} / \bullet$ & $\mathrm{LC} / \bullet$ & & & & & 1 & \\
\hline Bryum argenteum $\mathrm{Hedw}$. & $\mathrm{LC} / \bullet$ & $\mathrm{LC} / \bullet$ & 1 & & & & & \\
\hline Bryum caespitium Hedw. & $\mathrm{LC} / \bullet$ & $\mathrm{LC} / \bullet$ & 1 & & & & 1 & \\
\hline Bryum capillare Hedw. & $\mathrm{LC} / \bullet$ & $\mathrm{LC} / \bullet$ & & & 1 & & 1 & \\
\hline $\begin{array}{l}\text { Bryum pseudotriquetrum (Hedw.) } \\
\text { P.Gaertn. et al. }\end{array}$ & $\mathrm{LC} / \bullet$ & $\mathrm{LC} / \bullet$ & & & & 1 & & \\
\hline Bryum sp. & & & & 1 & & & 1 & \\
\hline $\begin{array}{l}\text { Calliergon cordifolium (Hedw.) } \\
\text { Kindb. }\end{array}$ & $\mathrm{LC} / \bullet$ & $\mathrm{LC} / \bullet$ & & & 1 & 1 & & \\
\hline $\begin{array}{l}\text { Calliergonella cuspidata (Hedw.) } \\
\text { Loeske }\end{array}$ & $\mathrm{LC} / \bullet$ & $\mathrm{LC} / \bullet$ & & & 1 & 1 & & 1 \\
\hline Ceratodon conicus (Hampe) Lindb. & $\mathrm{RE} / \mathrm{RE}$ & CR/CR & & & & & 1 & \\
\hline
\end{tabular}


Table 1 (continued)

\begin{tabular}{|c|c|c|c|c|c|c|c|c|}
\hline$\overline{\text { Species }}$ & $\begin{array}{l}\text { Status } \\
2011 \\
\end{array}$ & $\begin{array}{l}\text { Status } \\
2014 \\
\end{array}$ & $\begin{array}{l}\text { Tramp- } \\
\text { led area }\end{array}$ & $\begin{array}{l}\text { Dry } \\
\text { meadows }\end{array}$ & $\begin{array}{l}\text { Shore } \\
\text { meadows }\end{array}$ & Forests & Rocks & $\begin{array}{l}\text { Bogs, } \\
\text { fens }\end{array}$ \\
\hline Ceratodon purpureus (Hedw.) Brid. & $\mathrm{LC} / \bullet$ & $\mathrm{LC} / \bullet$ & 1 & 1 & 1 & & 1 & \\
\hline $\begin{array}{l}\text { Climacium dendroides (Hedw.) } \\
\text { F.Weber \& D.Mohr }\end{array}$ & $\mathrm{LC} / \bullet$ & $\mathrm{LC} / \bullet$ & 1 & & & & 1 & \\
\hline Dicranum leioneuron Kindb. & LC/RT & LC/RT & & & & & & 1 \\
\hline Dicranum polysetum Sw. ex anon. & $\mathrm{LC} / \bullet$ & $\mathrm{LC} / \bullet$ & & 1 & & & & \\
\hline Dicranum scoparium Hedw. & $\mathrm{LC} / \bullet$ & $\mathrm{LC} / \bullet$ & 1 & 1 & & 1 & 1 & 1 \\
\hline $\begin{array}{l}\text { Dicranum undulatum Schrad. ex } \\
\text { Brid. }\end{array}$ & $\mathrm{LC} / \bullet$ & $\mathrm{LC} / \bullet$ & & & & & & 1 \\
\hline $\begin{array}{l}\text { Ditrichum flexicaule (Schwägr.) } \\
\text { Hampe }\end{array}$ & $\begin{array}{l}\text { LC/ } \\
\text { IND }\end{array}$ & $\begin{array}{l}\text { LC/ } \\
\text { IND }\end{array}$ & 1 & & & & & \\
\hline $\begin{array}{l}\text { Drepanocladus aduncus (Hedw.) } \\
\text { Warnst. }\end{array}$ & $\mathrm{LC} / \bullet$ & $\mathrm{LC} / \bullet$ & & & & & 1 & \\
\hline $\begin{array}{l}\text { Drepanocladus polygamus (Schimp.) } \\
\text { Hedenäs }\end{array}$ & $\mathrm{LC} / \bullet$ & $\mathrm{LC} / \bullet$ & & & 1 & & & \\
\hline Fissidens adianthoides Hedw. & $\begin{array}{l}\text { LC/ } \\
\text { IND }\end{array}$ & $\begin{array}{l}\text { LC/ } \\
\text { IND }\end{array}$ & & & & 1 & & \\
\hline Fissidens osmundoides Hedw. & $\mathrm{LC} / \bullet$ & $\mathrm{LC} / \bullet$ & & & & & 1 & \\
\hline Hedwigia ciliata (Hedw.) P.Beauv. & $\mathrm{LC} / \bullet$ & $\mathrm{LC} / \bullet$ & & & & & 1 & \\
\hline Herzogiella seligeri (Brid.) Z.Iwats. & $\begin{array}{l}\text { LC/ } \\
\text { IND }\end{array}$ & $\begin{array}{l}\text { LC/ } \\
\text { IND }\end{array}$ & & & & 1 & & \\
\hline $\begin{array}{l}\text { Hylocomium splendens (Hedw.) } \\
\text { Schimp. }\end{array}$ & $\mathrm{LC} / \bullet$ & $\mathrm{LC} / \bullet$ & 1 & 1 & & & & \\
\hline Hypnum andoi A.J.E.Sm. & $\mathrm{LC} / \bullet$ & $\mathrm{LC} / \bullet$ & & & & 1 & & \\
\hline Hypnum cupressiforme Hedw. & $\mathrm{LC} / \bullet$ & $\mathrm{LC} / \bullet$ & 1 & 1 & & 1 & 1 & 1 \\
\hline Mnium hornum Hedw. & $\mathrm{LC} / \bullet$ & $\mathrm{LC} / \bullet$ & & & & 1 & 1 & \\
\hline Orthotrichum speciosum Nees & $\mathrm{LC} / \bullet$ & $\mathrm{LC} / \bullet$ & & & & 1 & & \\
\hline Philonotis fontana (Hedw.) Brid. & $\mathrm{LC} / \bullet$ & $\mathrm{LC} / \bullet$ & & & & & 1 & \\
\hline Philonotis tomentella Molendo & $\mathrm{LC} / \bullet$ & $\mathrm{LC} / \bullet$ & & & & & 1 & \\
\hline $\begin{array}{l}\text { Plagiomnium affine (Blandow ex } \\
\text { Funck) T.J.Kop. }\end{array}$ & $\mathrm{LC} / \bullet$ & $\mathrm{LC} / \bullet$ & & 1 & & & & \\
\hline $\begin{array}{l}\text { Plagiomnium cuspidatum (Hedw.) } \\
\text { T.J.Kop. }\end{array}$ & $\mathrm{LC} / \bullet$ & $\mathrm{LC} / \bullet$ & 1 & & & 1 & 1 & \\
\hline $\begin{array}{l}\text { Plagiomnium undulatum (Hedw.) } \\
\text { T.J.Kop. }\end{array}$ & $\begin{array}{l}\text { LC/ } \\
\text { IND }\end{array}$ & $\begin{array}{l}\text { LC/ } \\
\text { IND }\end{array}$ & & & & 1 & & \\
\hline $\begin{array}{l}\text { Plagiothecium denticulatum } \\
\text { (Hedw.) Schimp. }\end{array}$ & $\mathrm{LC} / \bullet$ & $\mathrm{LC} / \bullet$ & & & & 1 & & \\
\hline Plagiothecium latebricola Schimp. & NT/RT & NT/RT & & & & 1 & & \\
\hline $\begin{array}{l}\text { Pleurozium schreberi (Willd. ex } \\
\text { Brid.) Mitt. }\end{array}$ & $\mathrm{LC} / \bullet$ & $\mathrm{LC} / \bullet$ & & 1 & & & & 1 \\
\hline Pohlia nutans (Hedw.) Lindb. & $\mathrm{LC} / \bullet$ & $\mathrm{LC} / \bullet$ & 1 & 1 & & & & \\
\hline Pohlia sp. & & & & & & & & 1 \\
\hline $\begin{array}{l}\text { Polytrichastrum alpinum (Hedw.) } \\
\text { G.L.Sm. }\end{array}$ & $\mathrm{LC} / \mathrm{O}$ & $\mathrm{LC} / \bullet$ & & & & & 1 & \\
\hline $\begin{array}{l}\text { Polytrichastrum longisetum (Sw. ex } \\
\text { Brid.) G.L.Sm. }\end{array}$ & $\mathrm{LC} / \bullet$ & $\mathrm{LC} / \bullet$ & & & & 1 & & 1 \\
\hline
\end{tabular}


Table 1 (continued)

\begin{tabular}{|c|c|c|c|c|c|c|c|c|}
\hline Species & $\begin{array}{l}\text { Status } \\
2011 \\
\end{array}$ & $\begin{array}{l}\text { Status } \\
2014 \\
\end{array}$ & $\begin{array}{l}\text { Tramp- } \\
\text { led area }\end{array}$ & $\begin{array}{l}\text { Dry } \\
\text { meadows }\end{array}$ & $\begin{array}{l}\text { Shore } \\
\text { meadows }\end{array}$ & Forests & Rocks & $\begin{array}{l}\text { Bogs, } \\
\text { fens }\end{array}$ \\
\hline Polytrichum juniperinum Hedw. & $\mathrm{LC} / \bullet$ & $\mathrm{LC} / \bullet$ & 1 & & & & 1 & 1 \\
\hline Polytrichum piliferum Hedw. & $\mathrm{LC} / \bullet$ & $\mathrm{LC} / \bullet$ & & & & & 1 & \\
\hline Pylaisia polyantha (Hedw.) Schimp. & $\mathrm{LC} / \bullet$ & $\mathrm{LC} / \bullet$ & & & & 1 & & \\
\hline $\begin{array}{l}\text { Racomitrium heterostichum (Hedw.) } \\
\text { Brid. }\end{array}$ & $\mathrm{LC} / \bullet$ & $\mathrm{LC} / \bullet$ & & & & & 1 & \\
\hline $\begin{array}{l}\text { Rhodobryum roseum (Hedw.) } \\
\text { Limpr. }\end{array}$ & $\mathrm{LC} / \bullet$ & $\mathrm{LC} / \bullet$ & & 1 & & & & \\
\hline $\begin{array}{l}\text { Rhytidiadelphus squarrosus (Hedw.) } \\
\text { Warnst. }\end{array}$ & $\mathrm{LC} / \bullet$ & $\mathrm{LC} / \bullet$ & & 1 & & & & \\
\hline $\begin{array}{l}\text { Rhytidiadelphus triquetrus (Hedw.) } \\
\text { Warnst. }\end{array}$ & $\mathrm{LC} / \bullet$ & $\mathrm{LC} / \bullet$ & & 1 & & & & \\
\hline $\begin{array}{l}\text { Schistidium maritimum (Sm. ex } \\
\text { R.Scott) Bruch \& Schimp. }\end{array}$ & $\mathrm{LC} / \bullet$ & $\mathrm{LC} / \bullet$ & & & & & 1 & \\
\hline $\begin{array}{l}\text { Sciuro-hypnum oedipodium (Mitt.) } \\
\text { Ignatov \& Huttunen }\end{array}$ & $\mathrm{LC} / \bullet$ & $\mathrm{LC} / \bullet$ & & & 1 & 1 & & \\
\hline $\begin{array}{l}\text { Sciuro-hypnum populeum (Hedw.) } \\
\text { Ignatov \& Huttunen }\end{array}$ & $\mathrm{LC} / \bullet$ & $\mathrm{LC} / \bullet$ & & & & 1 & & \\
\hline $\begin{array}{l}\text { Sciuro-hypnum reflexum (Starke) } \\
\text { Ignatov \& Huttunen }\end{array}$ & $\mathrm{LC} / \bullet$ & $\mathrm{LC} / \bullet$ & & & & 1 & & \\
\hline $\begin{array}{l}\text { Sphagnum angustifolium } \\
\text { (C.E.O.Jensen ex Russow) } \\
\text { C.E.O.Jensen }\end{array}$ & $\mathrm{LC} / \bullet$ & $\mathrm{LC} / \bullet$ & & & & & & 1 \\
\hline $\begin{array}{l}\text { Sphagnum capillifolium (Ehrh.) } \\
\text { Hedw. }\end{array}$ & $\mathrm{LC} / \bullet$ & $\mathrm{LC} / \bullet$ & & & & & & 1 \\
\hline $\begin{array}{l}\text { Sphagnum fallax (H.Klinggr.) } \\
\text { H.Klinggr. }\end{array}$ & $\mathrm{LC} / \bullet$ & $\mathrm{LC} / \bullet$ & & & & 1 & & 1 \\
\hline Sphagnum fimbriatum Wilson & $\mathrm{LC} / \bullet$ & $\mathrm{LC} / \bullet$ & & & & & & 1 \\
\hline $\begin{array}{l}\text { Sphagnum fuscum (Schimp.) } \\
\text { H.Klinggr. }\end{array}$ & $\mathrm{LC} / \bullet$ & $\mathrm{LC} / \bullet$ & & & & & & 1 \\
\hline Sphagnum magellanicum Brid. & $\mathrm{LC} / \bullet$ & $\mathrm{LC} / \bullet$ & & & & & & 1 \\
\hline Sphagnum palustre L. & $\mathrm{LC} / \bullet$ & $\mathrm{LC} / \bullet$ & & & & 1 & & 1 \\
\hline Sphagnum papillosum Lindb. & $\mathrm{LC} / \bullet$ & $\mathrm{LC} / \bullet$ & & & & & & 1 \\
\hline Sphagnum riparium Ångstr. & $\mathrm{LC} / \bullet$ & $\mathrm{LC} / \bullet$ & & & & & & 1 \\
\hline Sphagnum rubellum Wilson & $\mathrm{LC} / \bullet$ & $\mathrm{LC} / \bullet$ & & & & & & 1 \\
\hline Sphagnum russowii Warnst. & $\mathrm{LC} / \bullet$ & $\mathrm{LC} / \bullet$ & & & & & & 1 \\
\hline Sphagnum squarrosum Crome & $\mathrm{LC} / \bullet$ & $\mathrm{LC} / \bullet$ & & & & & & 1 \\
\hline $\begin{array}{l}\text { Syntrichia ruraliformis (Besch.) } \\
\text { Cardot }\end{array}$ & NEW & $\begin{array}{l}\mathrm{DD} / \\
\mathrm{DD}\end{array}$ & 1 & & & & & \\
\hline $\begin{array}{l}\text { Syntrichia ruralis (Hedw.) F.Weber } \\
\text { \& D.Mohr }\end{array}$ & $\mathrm{LC} / \bullet$ & $\mathrm{LC} / \bullet$ & 1 & 1 & & & 1 & \\
\hline Warnstorfia fluitans (Hedw.) Loeske & $\mathrm{LC} / \bullet$ & $\mathrm{LC} / \bullet$ & & & & & & 1 \\
\hline
\end{tabular}

Liverworts

Calypogeia muelleriana (Schiffn.) LC/ LC/

Müll.Frib. IND IND

Cephalozia loitlesbergeri Schiffn. $\quad$ LC/O LC/ 
Table 1 (continued)

\begin{tabular}{|c|c|c|c|c|c|c|c|c|}
\hline$\overline{\text { Species }}$ & $\begin{array}{l}\text { Status } \\
2011 \\
\end{array}$ & $\begin{array}{l}\text { Status } \\
2014 \\
\end{array}$ & $\begin{array}{l}\text { Tramp- } \\
\text { led area }\end{array}$ & $\begin{array}{l}\text { Dry } \\
\text { meadows }\end{array}$ & $\begin{array}{l}\text { Shore } \\
\text { meadows }\end{array}$ & Forests & Rocks & $\begin{array}{l}\text { Bogs, } \\
\text { fens }\end{array}$ \\
\hline $\begin{array}{l}\text { Cephaloziella divaricata (Sm.) } \\
\text { Schiffn. }\end{array}$ & $\mathrm{LC} / \bullet$ & $\mathrm{LC} / \bullet$ & & 1 & & & 1 & \\
\hline $\begin{array}{l}\text { Lophocolea heterophylla (Schrad.) } \\
\text { Dumort. }\end{array}$ & $\mathrm{LC} / \bullet$ & $\mathrm{LC} / \bullet$ & & & & 1 & & \\
\hline Lophozia excisa (Dicks.) Dumort. & $\mathrm{LC} / \bullet$ & $\mathrm{LC} / \bullet$ & & & & & 1 & \\
\hline Mylia anomala (Hook.) Gray & $\mathrm{LC} / \bullet$ & $\mathrm{LC} / \bullet$ & & & & & & 1 \\
\hline Ptilidium ciliare (L.) Hampe & $\mathrm{LC} / \bullet$ & $\mathrm{LC} / \bullet$ & & & & & 1 & \\
\hline
\end{tabular}

moist soil in the borderline between a shore meadow and a flat rock. $B$. turgidum is now considered regionally threatened in Åland. Plagiothecium latebricola, a moss species that is nearly threatened in Finland and regionally threatened in Aland, grew on a stump of a deciduous tree in a moist forest patch. Dicranum leioneuron (least concern in Finland but regionally threatened in most of the country) grew between Sphagnum spp. in a small bog.

The species of Lågskär also included three species that are least concern in Finland but had not been observed in Åland after 1940: Brachythecium mildeanum (on bare mull in a moist forest patch), Polytrichastrum alpinum (in a small depression in a rock) and Cephalozia loitlesbergeri (among Sphagnum spp. in a small bog). In addition, the observed bryophytes include six species that indicate high nature values in their habitat: Brachythecium glareosum (in a dry, mown meadow and on trampled sandy soil between the buildings), Ditrichum flexicaule (shallow soil on a trampled path), Fissidens adianthoides (on mull in a moist, eutrophic forest patch), Herzogiella seligeri (on a spruce log in a forest with planted spruce trees), Plagiomnium undulatum (on mull in a moist forest patch) and Calypogeia muelleriana (among Sphagnum spp. in a small bog). These indicator species are mostly rare or declining species that have certain habitat requirements and their occurrence indicates that the habitat is likely to have biodiversity values (Ulvinen et al. 2002). For example, $B$. glareosum and $D$. flexicaule indicate calcareous soils.

\section{DISCUSSION}

The 81 species of Lågskär comprise $9 \%$ of all species found in Finland (a total of 920 species or subspecies, Sammaltyöryhmä 2014) or 14 $\%$ of the species found in Åland (577 species or subspecies, Sammaltyöryhmä 2014). Two of Lågskär's species, Syntrichia ruraliformis and Ceratodon conicus, were believed to be absent or extinct from mainland Finland as well as the other parts of Åland islands. Brachythecium turgidum was also believed to be extinct in Åland. In contrast, all of the species that were observed in Lågskär occur also in Sweden (Hallingbäck et al. 2006). Syntrichia ruraliformis, for example, occurs in southern Sweden wherefrom it could have spread to Lågskär. Alternatively, it could have been overlooked or misidentified as Syntrichia ruralis and therefore not recorded in Finland. This is supported by the fact that the species was reported in 2012 from a specimen that was collected in 2008 from an island in Parainen in the South-West corner of mainland Finland (Juutinen et al. 2013). Therefore it seems likely that the species occurs also elsewhere in the southern part of the country.

The bryophyte flora of Lågskär is limited by the small size of the island, the large distance from possible dispersal sources and the strong drying effects of wind and sun in most of the island. On the other hand, the species richness is increased by the diversity of habitats, the occurrence of meso- and eutrophic habitats and the human impacts (buildings, trampling, grazing and tree planting in the past, the management of the dry meadows). 
The occurrence of the 14 noteworthy species on Lågskär is due to the patchy calcareous substrates (Brachythecium glareosum, Brachythecium turgidum, Ceratodon conicus, Ditrichum flexicaule, Fissidens adianthoides, Plagiomnium undulatum), human impact (Brachythecium glareosum, Ceratodon conicus, Syntrichia ruraliformis), natural bogs and fens (Brachythecium turgidum, Dicranum leioneuron, Calypogeia muelleriana) and the natural moist herb-rich forests (Fissidens adianthoides, Herzogiella seligeri, Plagiomnium undulatum, Plagiothecium latebricola). The high number of species without recent observations in Åland (Brachythecium mildeanum, Brachythecium turgidum, Ceratodon conicus, Polytrichastrum alpinum, Syntrichia ruraliformis, Cephalozia loitlesbergeri) is most likely explained by the low amount of bryophyte inventories conducted on the Alland islands during the previous decades. Luckily bryophyte specialists have conducted several inventories on the islands during the last few years, and indeed, Brachythecium mildeanum has been reported by Syrjänen et al. (2013) and by Huttunen et al. (2014), and Polytrichastrum alpinum by Syrjänen et al. (2013). It is thus likely that many of the species of Lågskär occur also elsewhere in Åland.

\section{ACKNOWLEDGEMENTS}

I wish to thank the families Mikkola and Huttunen for the company on the island and Tauno Ulvinen, Riikka Juutinen and Hilja Vuori for helping with the identification of the most difficult specimens. I also want to thank two anonymous reviewers for valuable comments on the manuscript. The study was funded by Societas pro Fauna et Flora Fennica and microscopy facilities were provided by the Natural History Museum of the University of Jyväskylä.

\section{REFERENCES}

Ahti, T., Hämet-Ahti, L. \& Jalas, J., 1968. Vegetation zones and their sections in northwestern Europe. Annales Botanici Fennici 5: 169-211.
Hallingbäck, T., Hedenäs, L. \& Weibull, H. 2006. Ny checklista för Sveriges mossor (in Swedish with English summary). Svensk botanisk tidskrift 100: 96-148.

Huttunen, S., Ahonen, I., Bisang, I., Hedenäs, L., Laaka-Lindberg, S. \& Vänni, J. 2014. Sammalretki Ahvenanmaalle keväällä 2013 (in Finnish with English summary). Bryobrotherella 17: 114-135.

Juutinen, R., Ulvinen, T. \& Syrjänen, K. 2013. Suomen sammalien eliömaakunnallisen levinneisyysluettelon lisäyksiä ja poistoja 4 (In Finnish with English summary). Bryobrotherella 16: 84-93.

Laaksonen, J. 2004: Kevät lintusaarella - Luontopäiväkirja (in Finnish). Gummerus Kustannus Oy, Jyväskylä. 176 p.

Sammaltyöryhmä 2011. Sammalten levinneisyys metsäkasvillisuusvyöhykkeissä ja ELY-keskuksissa (in Finnish). Suomen ympäristökeskus. 30.5.2011. http: / / www.ymparisto.fi/fi-FI/ Luonto/Lajit/Lajiensuojelutyo/Eliotyoryhmat/ Sammaltyoryhma/Suomen_sammalet

Sammaltyöryhmä 2014. Sammalten levinneisyys metsäkasvillisuusvyöhykkeissä ja ELY-keskuksissa (in Finnish). Suomen ympäristökeskus. 6.3.2014. http: / / www.ymparisto.fi/fi-FI/Luonto/Lajit/ Lajiensuojelutyo/Eliotyoryhmat/Sammaltyoryhma/Suomen_sammalet

Syrjänen, K., Anttila, S., Ulvinen, T., Laaka-Lindberg, S., Huttunen, S., Laitinen, T., Ahonen, I., Fagerstén, R., He, X., Juslén, A., Korvenpää, T., Korvenpää, T., Parnela, A., Sallantaus, T., Vainio, O., Virtanen, R., Piippo, S. \& Rikkinen, J., 2010. Bryophytes. In: Rassi, P., Hyvärinen, E., Juslén, A. \& Mannerkoski, I. (eds). The 2010 Red List of Finnish Species. Ympäristöministeriö \& Suomen ympäristökeskus, Helsinki., pp. 208-230.

Syrjänen, K., Huttunen, S., Juutinen, R., Hedenäs, L., Bisang, I., Ahonen, I. \& Laaka-Lindberg, S. 2013: Sammalretki Ahvenanmaalle 17.-21.9.2012 (in Finnish with English summary). Bryobrotherella 16: $53-71$.

Ulvinen, T. \& Syrjänen, K., 2009. Suomen sammalten levinneisyys eliömaakunnissa. In: Laaka-Linberg, S., Anttila, S., Syrjänen, S. (eds). Suomen uhanalaiset sammalet (in Finnish with English summary). Suomen Ympäristökeskus, Helsinki, pp. 309-342.

Ulvinen, T., Syrjänen, K. \& Anttila, S. (toim.) 2002. Suomen sammalet - levinneisyys, ekologia, uhanalaisuus (In Finnish with English summary). The Finnish Environment 560. 
74 Folia Cryptog. Estonica 